\title{
Future Studies of Planetary Rings
} by Spaceprobes

\author{
E.C. Stone \\ California Institute of Technology \\ Pasadena, California 91125
}

SRL $83-30$

December 1983

A Chapter in "Planetary Rings", Brahic and Greenberg, eds., University of Arizona Press 


\author{
Future Studies of Planetary Rings by Spaceprobes \\ E.C. Stone \\ California Institute of Technology \\ Pasadena, California 91125
}

\begin{abstract}
Recent observations by the Pioneer and Voyager missions have been the basis for major advances in our knowledge and understanding of the Jovian and Saturnian rings. Future spaceprobe observations offer further opportunities for studying the ring systems of giant planets.
\end{abstract}

The first spaceprobe observations of planetary rings were carried out by the Voyager and Pioneer missions to Jupiter and Saturn. Even though limited in time by the brevity of planetary flybys, the missions nevertheless returned a wealth of information about planetary rings which is unobtainable by other means and which in many instances was completely unexpected. As a result, those observations have already had a major impact on our understanding of ring systems as is apparent in many chapters in this book.

This chapter describes further opportunities for ring studies by spaceprobes, including the Voyager 2 flybys of Uranus and Neptune, the Galileo mission to Jupiter, and a possible Saturn Orbiter mission. The results anticipated from these new studies have been inferred from the results obtained from the Voyager and Pioneer encounters with Jupiter and Saturn and are discussed in that context. 
The Voyager 2 encounter with Uranus in 1986 is a continuation of the exploratory mission to the outer Solar System that began in 1977 with the launch of two essentially identical spacecraft. As shown in Figure 1, Voyager 1 encountered Jupiter in March 1979, returning numerous new observations of that planetary system, including the first image of the Jovian ring as described in Science (204, 945-1007, 1979). Voyager 2 follcwed closely behind, providing additional observations of the Jovian system in July 1979 (Sctence, 206, 925-995, 1979).

Using the gravity assist of the Jovian flybys, both spacecraft continued on to Saturn, Voyager 1 arriving first in November 1980. Among the numerous discoveries about the Saturn system reported in Science (212. 159-243, 1981) were many new aspects of Saturn's rings, including "spokes", satellite-driven spiral density waves, hundreds of features in the B-ring, two small satellites confining the kinked, multistranded F-ring, and the size distribution of particles with radii ranging from several centimeters to 10 meters.

Following nine months later, Voyager 2 returned additional key observations of the Saturnian system (see Science, 215, $499-594,1982$ ), including a stellar occultation which provided measurements of the optical thickness of Saturn's rings with a radial resolution of $\leq 300 \mathrm{~m}$. These data indicated the existence of numerous spiral density waves excited by orbital resonances with several of the innermost satellites. It was also 
found that the outer edge of the B-ring is elliptical as expected from a 2:1 resonant interaction with Mimas.

Voyager 2 used the gravity assist of the Saturn flyby to continue on to Uranus and Neptune. Many of the techniques developed for the study of Saturn's rings will be employed during the Uranus encounter in January 1986, including stellar and radio occultations and high resolution imaging as described below.

At Saturn, studies of the rings and associated phenomena were carried out by many of the eleven scientific investigations on the voyager spacecraft. The locations of the instruments are shown in Figure 2, which also depicts several of the major engineering subsystems. A more comprehensive description of the eleven investigations listed in Table 1 will be found in Space Sclence Reviews (21, 75-376, 1977).

Although not explicitly noted in Figure 2, the four remote sensing instruments, ISS, IRIS, PPS, and UVS are mounted on a scan platform with two axes of articulation, permitting the instruments to be pointed at essentially any target. 
Stellar and Solar Occultation Studies

Since the discovery of the Uranian rings during a stellar occultation observation in 1977, subsequent occultation studies have provided detalled information on the rings' optical depths, eccentricities, precession, and Inclination (see, e.8., Elliot, et al., 1981, and references therein). Although Voyager 2 observations will add just a few additional stellar occultations to those already observed from Earth, they will provide much higher spatial resolution than Earth-based observations at $2.2 \mu \mathrm{m}$ that are limited by a Fresnel zone width of $3.5 \mathrm{~km}$. In contrast, the voyager 2 spatial resolution will be limited primarily by the instrument sampling times ( $10 \mathrm{~ms}$ for PPS, $320 \mathrm{~ms}$ for UVS) and transient response, since the Fresnel zone width is $\leq 15 \mathrm{~m}$. As a result, the spatial resolution expected at Uranus should be similar to that achieved at Saturn, where the photopolarimeter provided $110 \mathrm{~m}$ resolution and the ultraviolet spectrometer 3 km resolution. As at Saturn, comparable resolution of the vertical distribution of ring material is provided by detailed observations of the abruptness of the occultation at the edges of the rings.

Several stars have been identified as candidates for occultation studies during the Uranus encounter. Among these are Sigma Sagitarii and Epsilon Persei. The first of these occultations occurs while the instruments are viewing the illuminated face of the rings $\left(25^{\circ}\right.$ phase angle), the second occurs while viewing the other face ( $140^{\circ}$ phase angle). Figures 3 and 4 illustrate the viewing geometries for these two stellar occultations. Since scattered sunlight from the rings will ultimately 
limit the measurements of regions of low optical depth, observations at several phase angles are of particular value and additional stellar occultations may be included in the sequence of observations.

It may also be possible to perform a solar occultation study of the rings using the ultraviolet spectrometer. However, this study will have to take into account both the scattering of sunlight from ring particles in the spectrometer's field of view and the finite size of the sun which corresponds to a distance of $\sim 80 \mathrm{~km}$ on the ring plane. 


\section{Radio Occultation Studies}

Radio occultation studies provide information on the radial variation of the microwave opacity of the rings and on the scattering properties of ring particles. Thus, measurements of the attenuation of the coherent 3.6 and $13 \mathrm{~cm}$ radio waves which are transmitted through the rings by the spacecraft yield information on the number of ring particles with racil $\gtrsim 1$ and $\gtrsim 4 \mathrm{~cm}$, respectively. Simultaneous measurements of the angular distribution of the forward scattering by the ring particles yield information on the number distribution of larger ring particles with radii between 1 and 15 m (see for example, Tyler, et al., 1983, and Marouf, et al., 1983, and references therein for results from the Voyager 1 radio occultation study of Saturn's rings).

The occultation geometry for the Uranus flyby is 1llustrated in Figure 5 and summarized in Table 2. The spatial resolution of radial opacity variations is related to the size of the Fresnel zone. Due to the oblique passage of the radio beam through Saturn's rings, the radial component of the Fresnel zone was $\sim 20 \mathrm{~km}$ at $3.6 \mathrm{~cm}$ and $\sim 40 \mathrm{~km}$ at $13 \mathrm{~cm}$. However, the radio signals are coherent and the use of an inverse Fresnel transform resulted in a radial resolution of 75 to $300 \mathrm{~m}$ (Marouf and Tyler, 1983) in the F-ring and C-ring. Because the radio beam is essentially normal to the Uranian ring plane, the radial component of the Fresnel zone will be only $\sim 3$ and $\sim 6 \mathrm{~km}$ at the two wavelengths, so that subkilometer radial resolution should also be achievable. 
Another iujortant factor in determining the expected radial resolution is the signal level recorded at Earth, since a weaker signal requires a longer integration time in order to obtain a given signal-to-noise ratio. Although the signal intensity from Uranus' distance will be only $\sim 1 / 4$ of that from Saturn's, arraying of ground-based antennas will essentially double the receiving aperture so that signal integration time at Uranus w1ll only have to be double that at Saturn. Fortunately, the apparent radial velocity of the radio beam footprint in the Uranian ring plane is only $\sim 8 \mathrm{~km} / \mathrm{s}$, about $1 / 10$ that at Saturn where the beam obliquely penetrated the ring plane. As a result, better radial resolution ( $<1 \mathrm{~km}$ ) should be achieved at Uranus than at Saturn. 
Imaging Studies

Imaging has been particularly important in both the initial Voyager studies of the Jovian and Saturian rings (Smith, et al., 1979, 1980, 1981, 1982) and in subsequent detailed studies (e.g., Jewitt and Danielson, 1981, Cuzz1, etal., 1981, Lissauer, 1982, Esposito, et al., 1983, Por co, 1983, Shu, et al., 1983, and other chapters in this book). Although the imaging conditions will be difficult at Uranus, both because the ring albedo is low and because of the reduced solar illumination, there are a number of important observations anticipated.

Among the most important observations is a systematic search for small satellites associated with the ring system, such as the shepherding satellites proposed by Goldreich and Tremaine (1979). An inventory of the objects orbiting Uranus and an accurate determination of their orbital elements are essential to understanding the dynamics of the ring system. Even with the reduced illumination, objects the size of the F-ring shepherds ( $1980 \mathrm{~S} 26$ and $1980 \mathrm{~S} 27$ ) with diameters of $\sim 100 \mathrm{~km}$ should be easily detected, with the smallest detectable objects of unit albedo having diameters $<10 \mathrm{~km}$.

There will also be several sequences of images of the rings, including sequences at low phase angle taken on the approach to Uranus, a sequence in which the ring is imaged in front of the illuminated planet, and sequences taken at high phase angle after closest approach. The expected imaging capability is perhaps best illustrated by considering images taken of the 
eccentric ringlet in the Maxwell gap at $1.45 \mathrm{R}_{\mathrm{S}}$ in Saturn's rings. As discussed by Esposito, et al. (1983) and Porco (1983), this ring is structurally and dynamically similar to Uranus' $\varepsilon$-ring. The tworings have similar optical depths ( 1 to 2 ), similar surface mass densities ( $\left.20 \mathrm{~g} / \mathrm{cm}^{2}\right)$, and a similar range of radial widths ( 20 to $\left.\sim 100 \mathrm{~km}\right)$. One significant difference, however, is that the single particle albedo of Uranus' rings (Mathews, et al., 1982) is only 1/8 of that of the Maxwell ringlet (Esposito, et al., 1983). Taking into account differences in solar intensity and incidence angles, the $\varepsilon-$ ring will be $\sim 1 / 10$ as bright as the Maxwell ringlet.

Figure 6 is a Voyager 2 image of the C-ring showing the Maxwell gap and ringlet. This image was taken with the narrow angle camera, a clear filter, and a 0.72 second shutter. A similar image of the $\varepsilon$-ring would require a ten-times longer exposure. Although the spatial resolution of such a long exposure would usually be smear-limited to $\sim 30 \mathrm{~km} / \mathrm{line}$ pair, some of the images may have much better resolution when the residual spacecraft motion is particularly low. The faster wide angle camera with a geometrical resolution of $\sim 25 \mathrm{~km} / 1$ ne pair at 3 hours before closest approach will not be smear-limited.

Although the rings will be relatively dark in backscattered light, they may be considerably brighter when imaged at high phase angles if they contain significant numbers of micron-sized particles. Several wide angle images can be obtained at $170^{\circ}$ phase angle with a spatial resolution of $30 \mathrm{~km} / 1$ ine pair while the spacecraft is in the shadow behind Uranus. At 
Jupiter, narrow angle images taken at $174^{\circ}$ to $176^{\circ}$ phase angle displayed not only the ring with an optical depth $\tau \sim 3 \times 10^{-5}$, but also a diffuse halo of particles with $\tau \sim 7 \times 10^{-6}$. Although the illumination at Uranus is only 0.07 of that at Jupiter, the use of the wide angle camera, which is 9 times faster than the narrow angle, will allow direct imaging of very low optical depth rings of micron-sized particles which are undetectable by stellar or radio occultations. 
Trapped Radiation and Other Studies

Rings can also be detected by their absorption of trapped particles, provided that Uranus has a magnetosphere with stably-trapped energetic particles. As shown in Figure 7, detection of absorption features by the Low Energy Charged Particle Experiment and the Cosmic Ray System will be limited by the spacecraft trajectory to regions outside of $\sim 4.5 R_{U}$, assuming a magnetic dipole axis that is generally aligned with the planetary spin axis.

Al though there are no optically thick rings in the region beyond 4.5 $R_{U}$, there might be tenuous rings $(\tau<0.01)$ which are undetectable by Earth-based stellar occultations. Such tenuous rings can produce absorption signatures, as did the Jovian ring $\left(\tau \sim 3 \times 10^{-5}\right)$ (see, e.g., Acuña and Ness, 1976, Ip, 1979, and Pyle, et al., 1983) and the Saturnian G-ring $\left(\tau \sim 10^{-4}\right)$ (see, e.g., Simpson, et al., 1980, Van Allen, et al., 1980, Vogt, et al., 1982, and Van Allen, 1982, 1983). As discussed by Thomsen and Van Allen (1979) and Van Allen (1982, 1983), charged particle measurements not only provide information on the existence of tenuous rings, but also on the optical depth of the ring and on the effective size of the ring particles. For example, Van Allen found that the particulates in Saturn's G-ring have an effective radius $r=\left\langle r^{3}\right\rangle /\left\langle r^{2}\right\rangle$ in the range $0.035 \leq r \leq 0.1 \mathrm{~cm}$

The Plasma Wave and the Planetary Radio Astronomy instruments can also detect the electrical signals generated by the impact of ring particles as 
the spacecraft penetrates the ring plane. Such signals were detected at Saturn as Voyager 2 penetrated the ring plane just outside of the G-ring (Scarf, et al., 1982, and Warwick et al., 1982). Gurnett, et al. (1983) interpreted the signals as resulting from the impact of submicron-sized particles with an optical depth of $\sim 10^{-4}$. The instruments will have a similar sensitivity to any tenuous material at the location where voyager 2 penetrates the Uranian ring plane. 
THE NEPTUNE ENCOUNTER

With the gravity assist of the Uranian flyby, Voyager 2 will continue on to an encounter with Neptune, arriving there on August $24,1989$. Although Earth-based stellar occultations indicate an absence of optically thick rings at Neptune, tenuous rings, such as the Jovian ring or Saturn's G-ring, are not excluded by present observations. Thus, the techniques described above for detecting tenuous rings may provide evidence for previously undetected distributions of particles in orbit about Neptune.

The Neptune flyby trajectory, illustrated in Figure 8, was chosen to provide a close flyby of Neptune $\left(1.3 R_{N}\right)$ and $\operatorname{Triton}(\sim 44000 \mathrm{~km})$ and opportunities for radio and solar occultation studies of the atmospheres of both bodies. The resulting trajectory limits a solar (or radio) occultation study to regions beyond $\sim 1.6 \mathrm{R}_{\mathrm{N}}$. Although occultation studies are not optimum for detecting tenuous rings, limits on the particle size distribution can be obtained as was done for the Jovian ring (see e.g., Tyler, et al., 1981).

As at Jupiter and Saturn, other techniques can be employed to search for tenuous rings, Specifically, an edge-onview of the ring plane will occur as the spacecraft passes inbound through Neptune's equatorial plane at $3.66 R_{N}\left(1 R_{N}=24300 \mathrm{~km}\right)$, and a high phase angle view will be available as the spacecraft passes through Neptune's shadow. The Jovian ring, with an optical depth $\sim 3 \times 10^{-5}$ was visible from both such viewing geometries. An even more tenuous halo, with $\sim 7 \times 10^{-6}$, was visible in forward- 
scattered light. Thus, even with the reduced illumination at Neptune, it should be possible to detect a tenuous ring of micron-sized particles with $\tau \gtrsim 10^{-3}$. A stellar occultation study with a sufficiently bright star may also provide measurements of tenuous rings with $\tau \geqslant 10^{-2}$.

If Neptune has a magnetic field and stable trapped particles, the flyby trajectory will provide the opportunity to observe absorption signatures of ring particles beyond $\sim 2.45 \mathrm{R}_{N}$, assuming the axis of the magnetic field is aligned with Neptune's rotation axis. The inbound and outbound ring plane crossings also provide in situ detection of small ring particles by the Plasma Wave and Planetary Radio Astronomy instruments.

With the flyby of Neptune, the Voyager spacecraft will have completed their investigations of the rings of the giant outer planets. The Gallieo Mission to Jupiter will continue studies of the Jovian ring, while further spaceprobe studies of Saturn's rings await a new mission to place a properly instrumented spacecraft into orbit about Saturn. 
THE GALILEO MISSION TO JUPITER

The Galileo Mission to Jupiter is scheduled to be launched in 1986 and to arrive at Jupiter in 1988. The spacecraft, shown in Figure 9, will eject an atmospheric probe prior to being inserted into orbit about Jupiter. Although ring studies are not a primary objective of the mission, both the probe and the orbiter carry instruments that can provide new information on the ring. Table 3 contains a listing of all the Galileo instruments that are described in more detail in the Galileo Science Requirements Document (JPL document 625-50). An overview of the entire mission, its scientific objectives, and the characteristics of the orbiter and probe are provided by Johnson and Yeates (1983).

Most of the ring observations w1ll be made by instruments on the orbiter. After injection at radial distance of $4 R_{J}$ and an orbital inclination of $\sim 5^{\circ}$, subsequent close encounters with the Galilean satellites are used to raise perijove to $\gtrsim 9 \mathrm{R}_{\mathrm{J}}$ and to reduce the orbital inclination to a few tenths of a degree. Twelve orbits over a 20-month period will provide opportunities for ring plane crossing studies and nearly edge-on views of the ring over a complete range of phase angles. Among the instruments on the orbiter that may provide new information on the rings are the Solid State Imaging System (SSI), the Ultraviolet Spectrometer (UVS), and the Dust Detect or (DDS). 
The imaging system on the Gallleo orbiter uses a spare Voyager optical system with $1500 \mathrm{~mm}$ focal length, with the Voyager vidicon sensor replaced by an $800 \times 800$ pixel charge-coupled device (CCD) which is more than 25 times faster, a particular advantage in 1 maging the tenuous Jovian ring. The inherent photometric accuracy of the CCD will facilitate a study of the phase angle dependence of the scattering properties of the ring particles for wavelengths between 0.42 and $1.1 \mu \mathrm{m}$, potentially providing information on the size distribution of micron-sized particles.

The long observing time will also permit a much more accurate determination of the orbital elements of Metis and Adrastea and a search for other small satelites that may both supply ring material and dynamically control its spatial distribution. At closest approach to the ring, the spatial resolution of the camera will be $\sim 10 \mathrm{~km} / \mathrm{line}$ pair.

The Ultraviolet Spectrometer, operating in a wavelength range between 1150 and 4300 angstroms with sampling times down to $1 \mathrm{~ms}$, may also provide significant new information on the Jovian ring. The sampling times correspond to a spatial sampling of < $100 \mathrm{~m}$. Al though the instrument sensitivity is limited to normal optical depths of $>10^{-3}$, the low inclination of the orbit provides long slant paths through the tenuous ring. Thus, stellar occultation studies of the Jovian ring should be possible.

Although the orbiter remains well outside of the ring, the Dust Detector can make in situ measurements of small particles that may be more 
widely dispersed throughout the Jovian neighborhood. The detect or is sensitive to particles with masses between $10^{-6}$ and $10^{-16} \mathrm{~g}$, corresponding to particles with diameters ranging from $~ 0.02$ to $50 \mu \mathrm{m}$. Up to 100 particles with speeds from 2 to $50 \mathrm{~km} / \mathrm{s}$ can be analyzed each second.

The probe will also return information on the distribution of ring material as indicated by the absorption of trapped radiation. The probe contains a heavily shielded $\left(20.87 \mathrm{~g} / \mathrm{cm}^{2}\right)$ energetic particle detector providing measurements of the fluxes of electrons, protons, alpha particles, and heavier nuclei such as oxygen and sulfur. The detector will be turned on at $4 R_{J}$, returning flux measurements every $0.02 R_{J}$ as the probe plunges toward the Jovian atmosphere, providing some spatial resolution of the absorption signature of the bright ring which is $\sim 0.08$ $R_{J}$ in width. 


\section{A SATURN ORBITER MISSION}

Further spaceprobe studies of Saturn's rings await the placement of a properly instrumented spacecraft into an inclined orbit about Saturn. Such a mission could address questions about the macrostructure of the rings on the scale of $\gtrsim 10^{3} \mathrm{~km}$, the microstructure of the rings on smaller scales, the mass of the rings and the particle size distribution, the particle composition, and the electromagnetic properties of the ring material. The comprehensive longitudinal and temporal coverage provided by an orbiter would make possible a number of important studies which cannot otherwise be adequately addressed.

Among the key instruments for such studies would be a high speed, multiband photometer that could undertake multiple stellar occultation measurements with high spatial resolution. As has beenillustrated by Elliott, et al. (1981) for Uranus, multiple stellar occul ta tions provide important information on dynamically significant characteristics such as the density profile, eccentricity, and precession of the edges of gaps in the rings. Comprehensive imaging from an orbiter can also provide a detailed description of dynamically-controlled azimuthal variations in various ring structures (see, e.g., Porco, 1983) and waves (see, e.g., Shu,

et al., 1983). Imaging would also permit definitive searches for moonlets within the empty gaps.

An orbiting spacecraft also provides multiple opportunities for studying the physical thickness of the rings. Thus, the thickness of the 
ring at sharp edges can be estimated from the comparison of edge profiles acquired for different slant paths of the occulted starlight, with multiple radio occultations providing similar information for particles with diameters larger than $\sim 2 \mathrm{~cm}$. High speed photometry of the edge of the ring as the spacecraft passes through the ring plane can provide additional information on the apparent thickness of the rings.

Multiple radio occultations can also yield the size distribution of centimeter and meter sized particles throughout the ring system, especially if the rings are more open than during the Voyager encounter and therefore offer a smaller slant optical depth through the very dense B-ring. In addition, ring scattering experiments in which the spacecraft radio beam is scattered obliquely of the rings should allow a determination of the size distribution of particles with radil in the centimeter to meter range, complementing $\mathrm{X}$-band and S-band occul tation data that allow a determination of the distribution of particles with radii $r$ in the range $1 \leqslant r \leqslant 4 \mathrm{~cm}$ and $1 \leq r \leq 10 \mathrm{~m}$. It would also be of interest to consider approaches yielding information on the distribution of particles with $r>10 \mathrm{~m}$. It is possible, for example, that kilometer-sized particles create wakes in the ring that can be directly imaged or statistically sampled by multiple stellar and radio occultations.

Significant improvements in spatial resolution will also be possible with an increased signal-to-noise ratio resulting from greater transmitter power, larger collecting aperture on Earth, and improved occultation geometry. During the Voyager radio occultations, these factors resulted in 
radial resolutions of $300 \mathrm{~m}$ in the inner C-ring, $75 \mathrm{~m} \mathrm{in} \mathrm{the} \mathrm{F-ring,} \mathrm{and}$ several kilometers in the A-ring and parts of the B-ring (Marouf and Tyler, 1983). Since, in principle, the resolution is limited only by the size of the spacecraft antenna (typically $\sim 5 \mathrm{~m}$ ) significant improvements should be readily achievable. In addition, analysis of the near-forward scatter signal can provide a measure of the ring thickness in different regions of the rings (Zebker and Tyler, 1983).

Compositional studies with high spatial resolution would also be desirable. Such studies could be undertaken, for example, with an imaging spectrometer operating in the near infrared and a photopolarimeter similar to those on the Galileo orbiter.

With an orbiter it would also be possible to better characterize the electromagnetic properties of the spokes through high time resolution imaging of their radial and azlmuthal development and through longer term synoptic studies of their dependence on Saturnian longitude and local time. It would also be desirable to better determine the density of the hrdincen cloud associated with the ring. 
CONCLUSION

Recent spaceprobe observations of the rings of Jupiter and Saturn have provided an unparalleled increase in our knowledge and understanding of planetary rings. Employing many of the same observational techniques at Uranus and Neptune, Voyager 2 offers further opportunities for studying the nature of ring systems of giant planets. The Galileomission to Jupiter provides the first opportunity for long-term spaceprobe studies of a planetary ring system, offering the prospect of studies and discoveries beyond those accessible to the earlier Pioneer and Voyager flybys. A properly instrumented Saturn orbiter would not only provide a similar opportunity for studies of Saturn's rings, but is likely the only means by which it will be possible to adequately address the nature of the diverse phenomena displayed by this prototypical planetary ring system.

\section{ACKNOWLEDG EMENTS}

I appreciate helful comments by S.A. Collins, J.N. Cuzzi, J.B. Holberg, T.V. Johnson, A.L. Lane, and E.D. Miner. This work was partially supported by the National Aeronautics and Space Administration. 


\section{$\underline{\text { References }}$}

Acuna, M.H., and Ness, N.F. 1976. The main magnetic field of Jupiter. J. Geophysical Res, 81: 2917-2922.

Cuzzi, J.N., Lissauer, J.J., and Shu, F.H. 1981. Density waves in Saturn's rings. Nature 292: 703-707.

Elliot, J.L., French, R.G, Froge1, J.A., Elias, J.H., M ink, D.J., and Liller, W. 1981. Orbits of nine Uranian rings. Astron. J. 86: 444-455.

Esposito, L.W., Borderies, N., Cuzzi, J.N., Goldreich, P., Holberg, J.B., Lane, A.L., Lissauer, J.J., Marouf, E.A., Pomphrey, R.B., Terrile, R.J., Tyler, G.L. 1983. Voyager observations of an eccentric ringlet in Saturn's C-Ring. Submitted to Science.

Goldreich, P., and Tremaine, S. 1979. Towards a theory for the Uranian rings. Nature 277: 97-99.

Gurnett, D.A., Grun, E., Gallagher, D., Kurth, W.S., and Scarf, F.L. 1983. Micron-sized particles detected near Saturn by the Voyager 2 plasma wave instrument. Icarus 53: 236-254. 
Ip, W.H. 1979. On the Pioneer 11 observations of the ring of Jupiter. Nature 280: $478-479$.

Jewitt, D.C., and Danielson, G.E., 1981. The Jovian ring. J. Geophysica1 Res, 86: 8691-8698.

Johnson, T.V., and Yeates, C.M. 1983. Return to Jupiter: Project Galileo. Sky and Telescope 66: 99-106.

Lissauer, J.J. 1982. Dynamics of Saturn's rings. Ph.D. thesis, University of California, Berkeley.

Marouf, E.A., Tyler, G.L. 1983. Radio occultation of Saturn's rings: Is less than 75 meters resolution achievable? Bull. American Astronomical Soc. 15: 817

Marouf, E.A., Tyler, G.L., Zebker, H.A., Simpson, R.A., and Eshleman, V.R. 1983. Particle size distributions in Saturn's rings from Voyager 1 radio occultation. Icarus 54: 189-211.

Mat thews, K., Neugebauer, G., and Nicholson, P.D. 1982. Maps of the rings of Uranus at a wavelength of 2.2 microns. Icraus 52: $126-$ 135. 
Porco, C. 1983. Voyager observations of Saturn's rings 1. The eccentric rings at $1.29,1.45 .1 .95$, and $2.27 \mathrm{R}_{\mathrm{S}}$. 2. The periodic variation of spokes. Ph.D. thesis, California Institute of Technology.

Pyle, K.R., McKibben, R.B., and Simpson, J.A. 1983. Pioneer 11 observations of trapped particle absorption by the Jovian ring and the satellites 1979, J1, J2, J3. J. Geophysical Res. 88: 45-48.

Scarf, F.L., Gurnett, D.A., Kurth, W.S., and Poynter, R.L. 1982 . Voyager 2 plasma wave observations at Saturn. Science 215: 587-594.

Shu, F.H., Cuzzi, J.N., and Lissauer, J.J. 1983. Bending waves in Saturn's rings. Icarus 53: 185-206.

Simpson, J.A., Bastian, T.S., Chenette, D.L., Lentz, G.A., McKibben, R.B., Pyle, K.R., and Tuzzolino, A.J. 1980. Saturnian trapped radiation and its absorption by satellites and rings: the first results from Pioneer 11. Science 207: 411-415.

Smith, B.A., Soderblom, L.A., Johnson, T.V., Ingerso1l, A.P., Collins, S.A., Shoemaker, E.M., Hunt, G.E., Masursky, H., Carr, M.H., Davies, M.E., Cook, A.F., Boyce, J., Danielson, G.E., Owen, T., Sagan, C., Beebe, R.F., Veverka, J., Strom, R.G., McCauley, J.F., Morrison, D., Briggs, G.A., Suomi, V.E., 1979. The Jovian system through the eyes of Voyager 1. Science 204: 951-972. 
Smith, B.A., Soderblom, L.A., Beebe, R., Boyce, J., Briggs, G., Carr, M., Collins, S.A., Cook, A.F., Danielson, G.E., Davies, M.E., Hunt, G.E., Ingersol1, A., Johnson, T.V., Masursky, H., McCauley, J., Morrison, D., Owen, T., Sagan, C., Shoemaker, E.M., Strom, R., Suomi, V.E., Veverka, J. 1980. The Galilean satellites and Jupiter: Voyager 2 Imaging Science results. Science 206: 927-950.

Smith, B.A., Soderblom, L., Beebe, R., Boyce, J., Briggs, G., Bunker, A., Collins, S.A., Hansen, C.J., Johnson, T.V., Mitche11, J.L., Terrile, R.J., Carr, M., Cook, A.F., Cuzzi, J., Pollack, J.B., Danielson, G.E., Ingerso11, A., Davies, M.E., Hunt, G.E., Masursky, H., Shoemaker, E., Morrison, D., Owen, T., Sagan, C., Veverka, J., Strom, R., Suomi, V.E. 1981. Encounter with Saturn: Voyager 1 imaging science results. Science 212: 163-191.

Smith, B.A., Soderblom, L., Batson, R., Bridges, P., Inge, J., Masursky, H., Shoemaker, E., Beebe, R., Boyce, J., Briggs, G., Bunker, A., Collins, S.A., Hansen, C.J., Johnson, T.V., Mitche11, J.L., Terrile, R.J., Cook, A.F., Cuzzi, J., Pollack, J.B., Danielson, G.E., Ingersol1, A.P., Davies, M.E., Hunt, G.E., Morrison, D., Owen, T., Sagan, C., Veverka, J., Strom, R., Suomi, V.E. 1982. A new look at the Saturn system: The Voyager 2 images. Science 215: 504-537. 
Thomsen, M.F. and Van Allen, J.A. 1979. On the inference of properties of Saturn's Ring E from energetic charged particle observations. Geophys. Res. Letters 6: 893-896.

Tyler, G.L., Marouf, E.A., Simpson, R.A., Zebker, H.A., and Eshleman, V.R. 1983. The microwave opacity of Saturn's rings at wavelengths of 3.6 and $13 \mathrm{~cm}$ from Voyager 1 radio occultation. Icarus 54: 160188 .

Tyler, G.L., Marouf, E.A., and Wood, G.E. 1981. Radio occultation of Jupiter's ring: bounds on optical depth and particle size and a comparison with optical and infrared results. J. Geophysical Res. 86: $8699-8703$.

Van Allen, J.A. 1982. Findings on rings and inner satellites of Saturn by Pioneer 11. Icarus 51: 509-527, 1982.

Van Allen, J.A. 1983. Absorption of energetic protons by Saturn's Ring G. J.Geophysical Res, 88: 6911-6918.

Van Allen, J.A., Thomsen, M.F., Randa11, B.A., Rairden, R.L., and Grosskreutz, C.L. 1980. Saturn's magnetosphere, rings, and inner satellites. Science 207: 415-421. 
Vogt, R.E., Chenette, D.L., Cummings, A.C., Garrard, T.L., Stone, E.C., Schardt, A.W., Trainor, J.H., Lal, N. and McDona1d, F.B. 1982. Energetic charged particles in Saturn's magnetosphere: Voyager 2 results. Science 215: 577-582.

Warwick, J.W., Evans, D.R., Romig, J.H., Alexander, J.K., Desch, M.D., Kaiser, M.L., Aubier, M., Leblanc, T., Lecacheux, A., and Pedersen, B.M. 1982. Planetary radio astronomy observations from Voyager 2 near Saturn. Science 215: 582-587.

Zebker, H.A., and Tyler, G.L. 1983. Thickness of Saturn's rings from Voyager 1 observation of microwave scatter. Science. 
Table 1. Voyager Science Investigations

\begin{tabular}{ll}
\hline Investigation Team & Principal Investigator/Institution \\
\hline Imaging Science (ISS) & Smith/Univ. Arizona (Team Leader) \\
Infrared Spectroscopy and Radiometry (IRIS) & Hanel/GSFC \\
Photopolarimetry (PPS) & Lane/JPL \\
Ul traviolet Spectroscopy (UVS) & Broadfood/Univ. Arizona \\
Radio Science (RSS) & Tyler/Stanford Univ. (Team Leader) \\
Magnetic Fields (MAG) & Ness/GSFC \\
Plasma (PLS) & Bridge/MIT \\
Plasma Wave (PWS) & Scarf/TRW \\
Planetary Radio Astronomy (PRA) & Warwick/Radiophysics, Inc. \\
Low Energy Charged Particles (LECP) & Krimigis/JHU/APL \\
Cosmic Rays (CRS) & Vogt/Caltech \\
\hline
\end{tabular}


Table 2. Voyager 2 Uranus Encounter

\begin{tabular}{|c|c|c|}
\hline \multicolumn{2}{|c|}{$\begin{array}{l}\text { Time, Closest Approach } \\
\text { (Spacecraft Event Time, GMT) }\end{array}$} & January 24, 1986 18:00 \\
\hline \multicolumn{2}{|c|}{ Radius, Closest Approach } & $107,080 \mathrm{~km}$ \\
\hline \multicolumn{2}{|c|}{ Radius, Ring Plane Crossing } & $115,200 \mathrm{~km}$ \\
\hline $\begin{array}{l}\text { Distance }\left(10^{3} \mathrm{~km}\right) \\
\text { Earth Occul tation }\end{array}$ & $\left\{\begin{array}{l}\varepsilon \text { Ring } \\
\text { Ring } 6 \\
\text { Uranus entrance } \\
\text { Uranus exit } \\
\text { Ring } 6 \\
\varepsilon \text { Ring }\end{array}\right.$ & $\begin{array}{l}137 \\
155 \\
186 \\
255 \\
280 \\
298\end{array}$ \\
\hline $\begin{array}{l}\text { Distance }\left(10^{3} \mathrm{~km}\right) \\
\text { Sun Occultation }\end{array}$ & $\begin{array}{l}\text { Uranus entrance } \\
\text { Uranus exit }\end{array}$ & $\begin{array}{l}177 \\
240\end{array}$ \\
\hline
\end{tabular}


Table 3. Galileo's Scientific Payload

\begin{tabular}{|c|c|}
\hline Probe Experiment & Principal Investigator \\
\hline Atmospheric Structure Instrument (ASI) & Seiff/ARC \\
\hline Neutral Mass Spectrometer (NMS) & Niemann/GSFC \\
\hline Hel ium Abundance Detector (HAD) & VonZahn/Univ. of Bonn \\
\hline Nephel ometer (NEP) & Ragent/ARC \\
\hline Net-Flux Radiometer (NFR) & Boese/ARC \\
\hline Lightning and Energetic Particles (LRD/EPI) & Lanzerotti/Bell Labs \\
\hline \multicolumn{2}{|l|}{ Orbiter Experiment } \\
\hline Solid-State Imaging (SSI) & Belton/KPNO (Team Leader) \\
\hline Near-Infrared Mapping Spectrometer (NIMS) & Carlson/JPL \\
\hline Ul traviolet Spectrometer (UVS) & Hord/Univ. of Colorado \\
\hline Photopolarimeter-Radiometer (PPR) & Hanssen/Goddard Inst. for Space Studies \\
\hline Magnetometer (MAG) & Kivel son/UCLA \\
\hline Energetic-Particle Detector (EPD) & Williams/APL \\
\hline Plasma Detector (PLS) & Frank/Univ. of Iowa \\
\hline Plasma-Wave Spectrometer (PWS) & Gurnett/Univ. of Iowa \\
\hline Dust Detector (DDS) & Grun/Max-Planck Inst. \\
\hline Radio Science (RS): Celestial Mechanics & Anderson/JPL (Team Leader) \\
\hline Radio Science (RS): Propagation & Howard/Stanford (Team Leader) \\
\hline
\end{tabular}




\section{Figure Captions}

Figure 1. A view normal to the ecliptic plane of the trajectories of Voyager 1 and 2 .

Figure 2. A drawing of the Voyager spacecraft showing the locations of the science instruments and several spacecraft subsystems. The Radio Science investigation uses the high gain antenna, the spacecraft $X-$ and $S-b a n d ~ t r a n s m i t t e r s$, and an ultrastable oscillator.

Figure 3. A view from Voyager 2 of Uranus and its rings during an occultation of Sigma Sagitarii. The instruments will be viewing the illuminated face of the rings.

Figure 4. A view from Voyager 2 of Uranus and its rings during an occultation of Epsilon Persei. The instruments will be viewing the unilluminated face of the rings.

Figure 5. A view normal to the plane of the trajectory of the Voyager 2 Uranus encounter on January 24, 1986, illustrating the intervals during which Earth (GEOCC) and solar (SUNOCC) occultations occur. 
Figure 6. A Voyager 2 image of the eccentric ringlet in the Maxwell Gap at $1.45 \mathrm{R}_{\mathrm{S}}$, taken from a range of $542,000 \mathrm{~km}$. There are many similarities between this ringlet and the $\varepsilon$-Ring at Uranus. However, particles of the $\varepsilon$-Ring have a much smaller albedo.

Figure 7. A representation of the Voyager 2 trajectory through a hypothetical magnetic field with the dipole axis aligned with the rotation axis of Uranus. In this hypothetical case the spacecraft would not cross magnetic field lines threading the known rings, but would be limited to the detection of absorption signatures of matter beyond $4.45 \mathrm{R}_{\mathrm{U}}$.

Figure 8. A view normal to the plane of the trajectory of the Voyager 2 encounter with Neptune on August 24, 1989, illustrating the periods during which Earth (GEOCC) and solar (SUNOCC) occultations occur.

Figure 9. A drawing of the Galileo spacecraft showing the locations of the science instruments and several spacecraft subsystems. The probe also carries a number of instruments. 


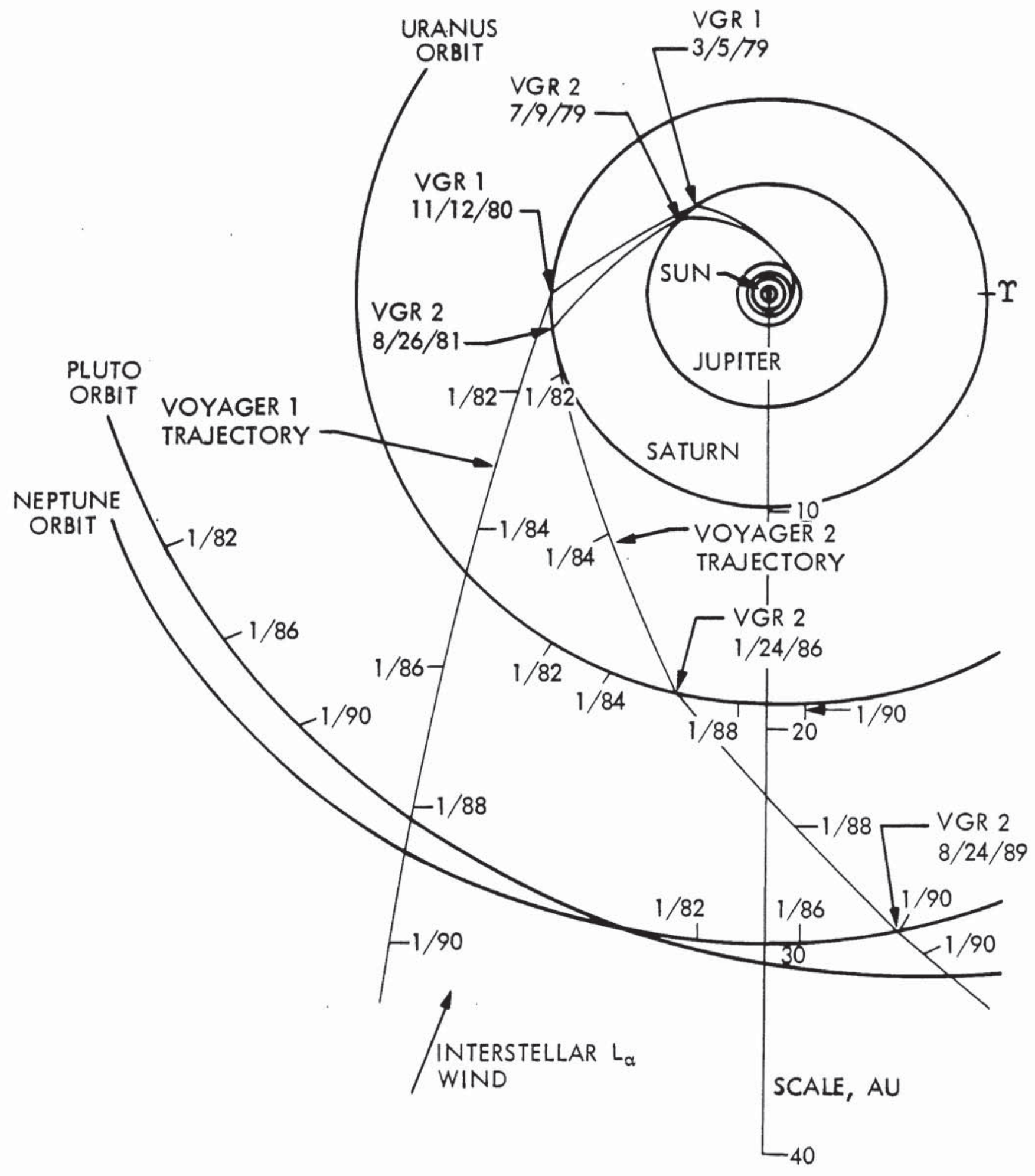

Figure 1 


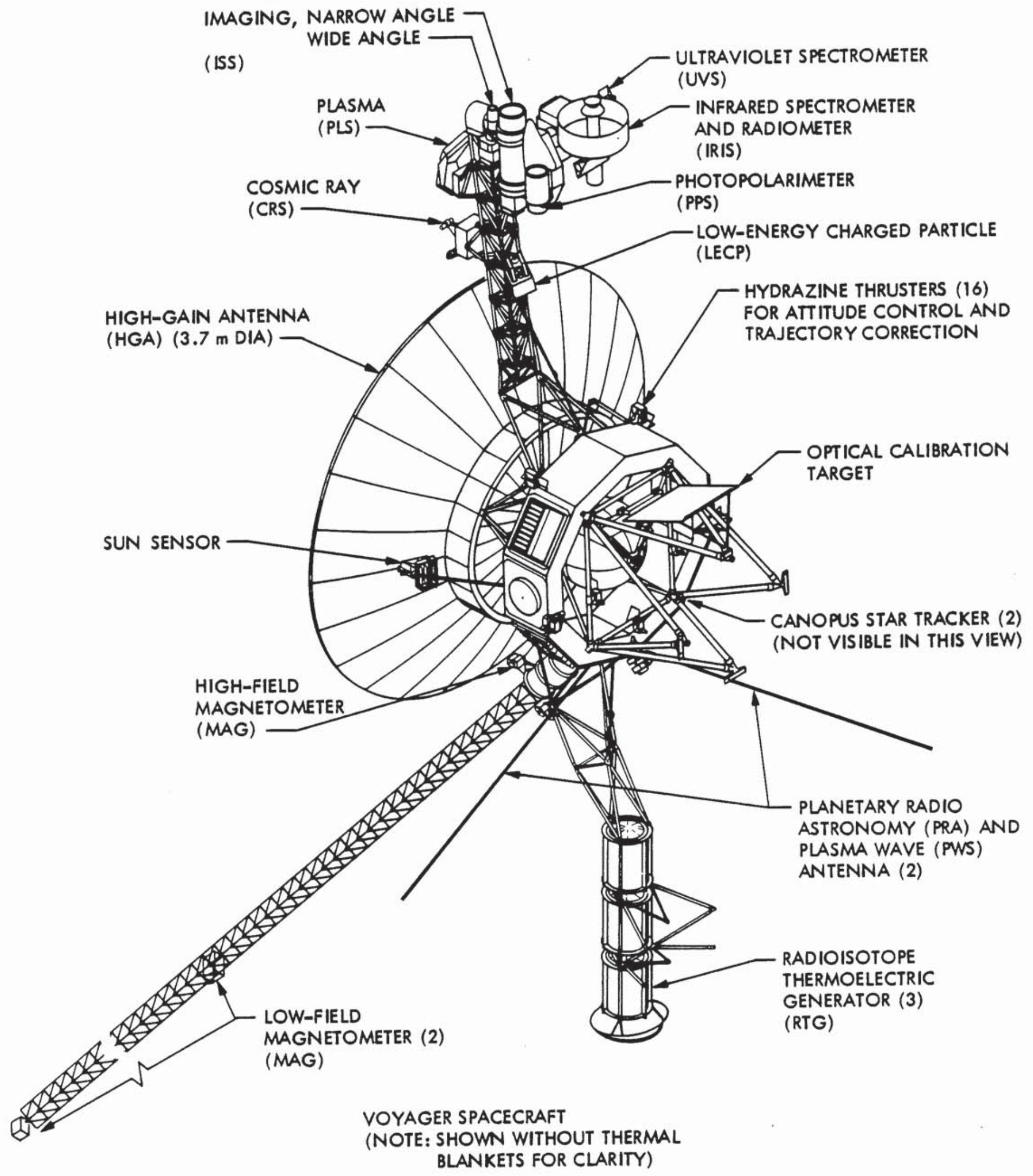

Figure 2 


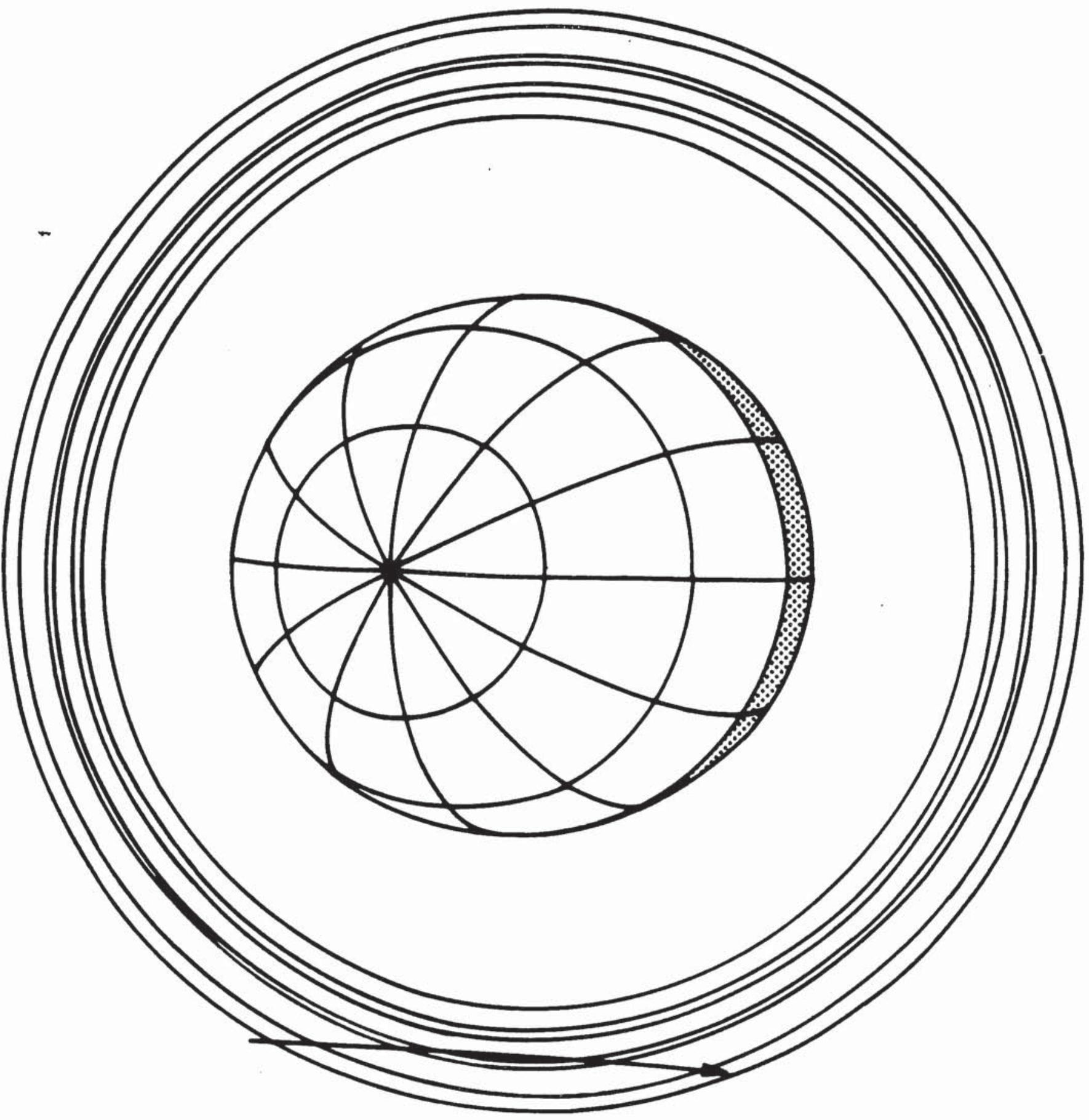

Figure 3 


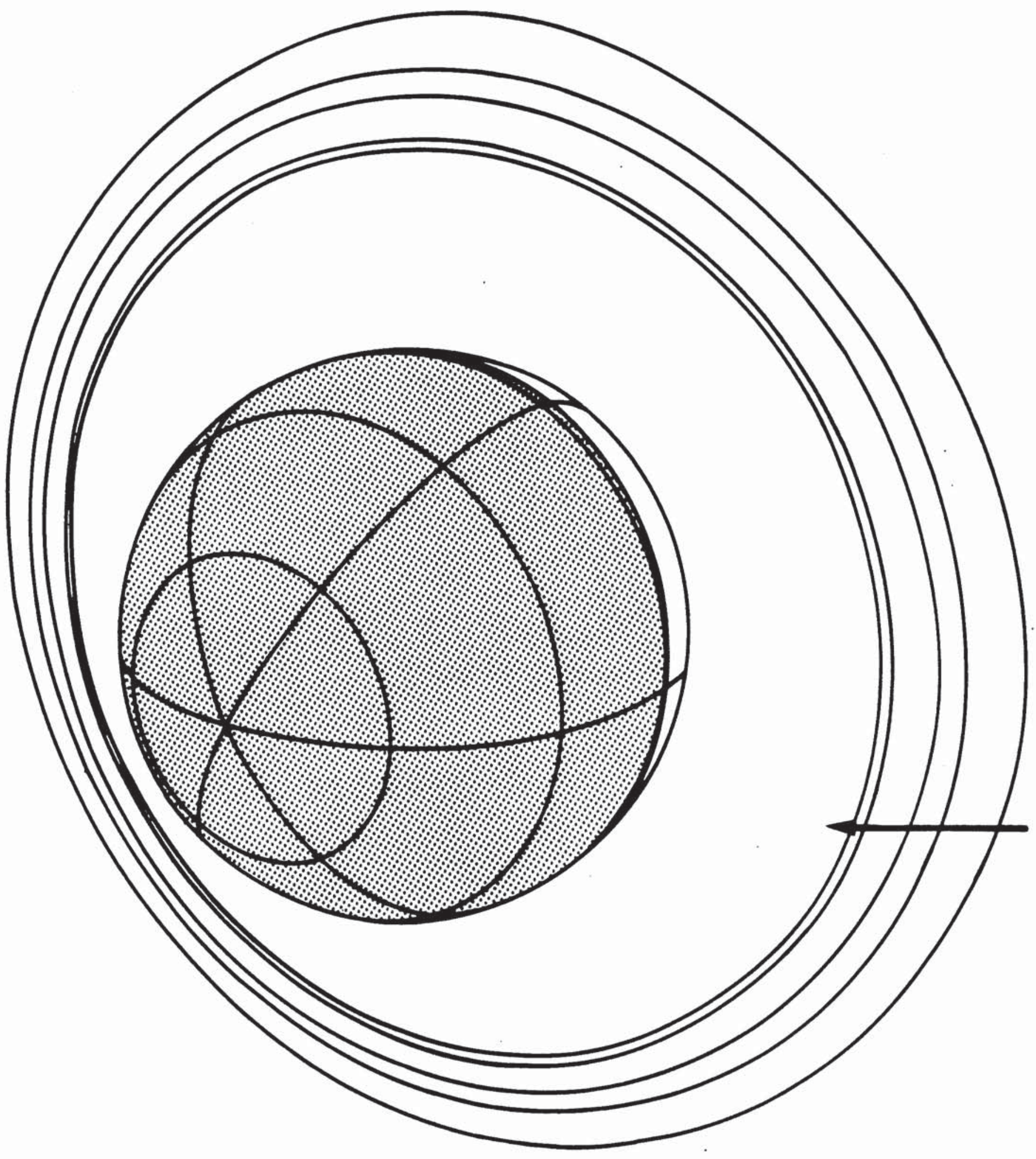

Figure 4 


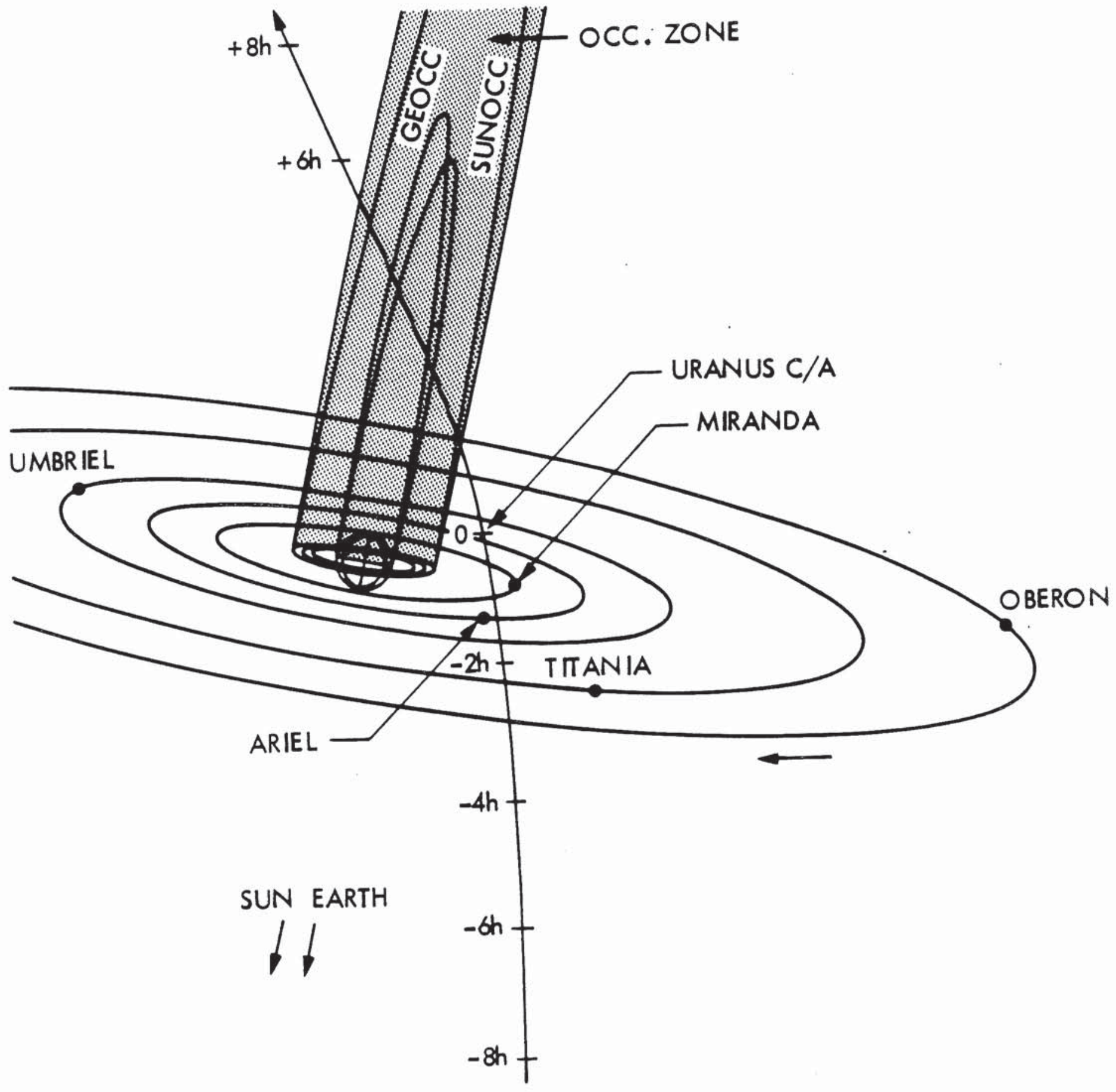

Figure 5 


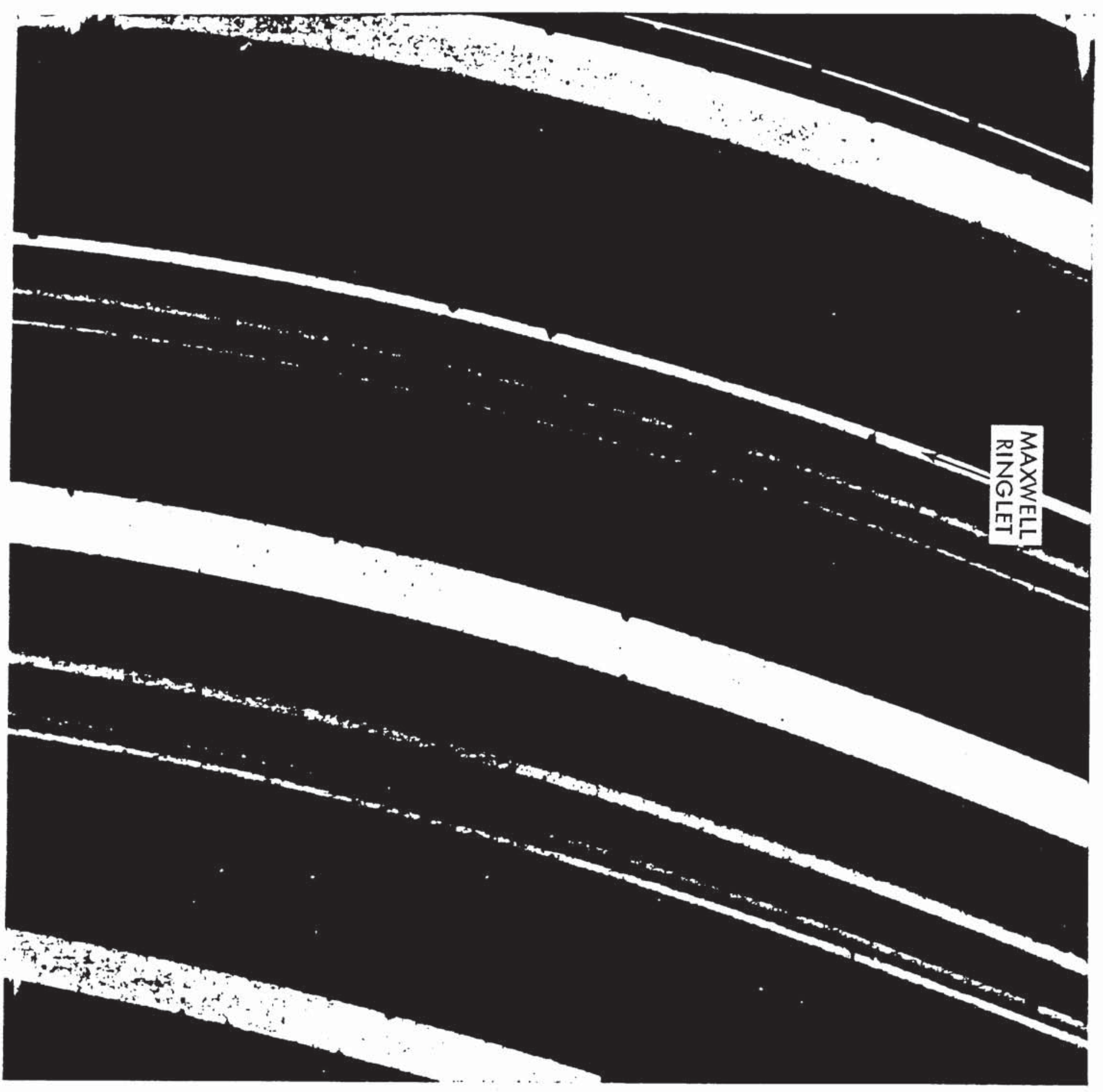

Figure 6 


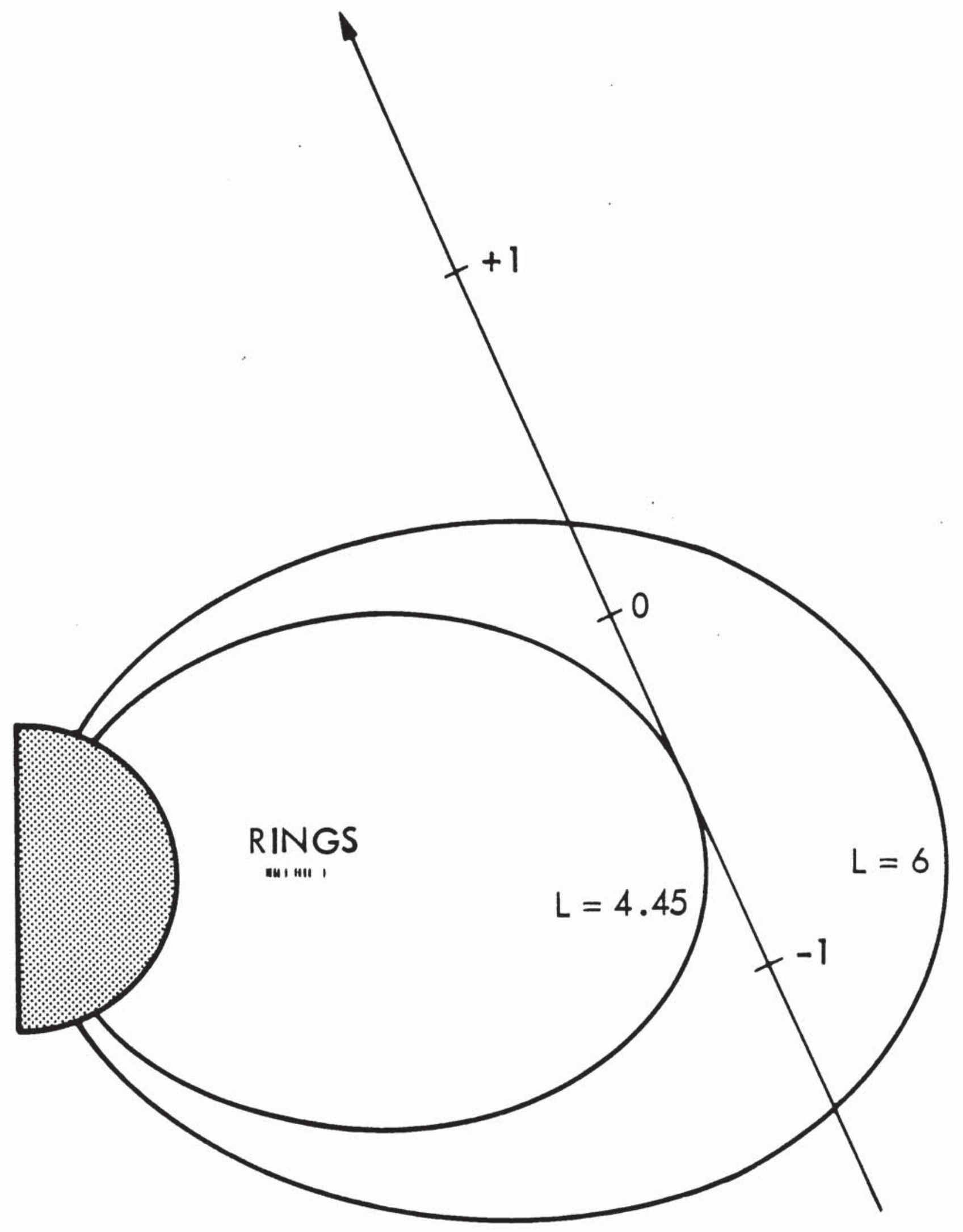

Figure 7 


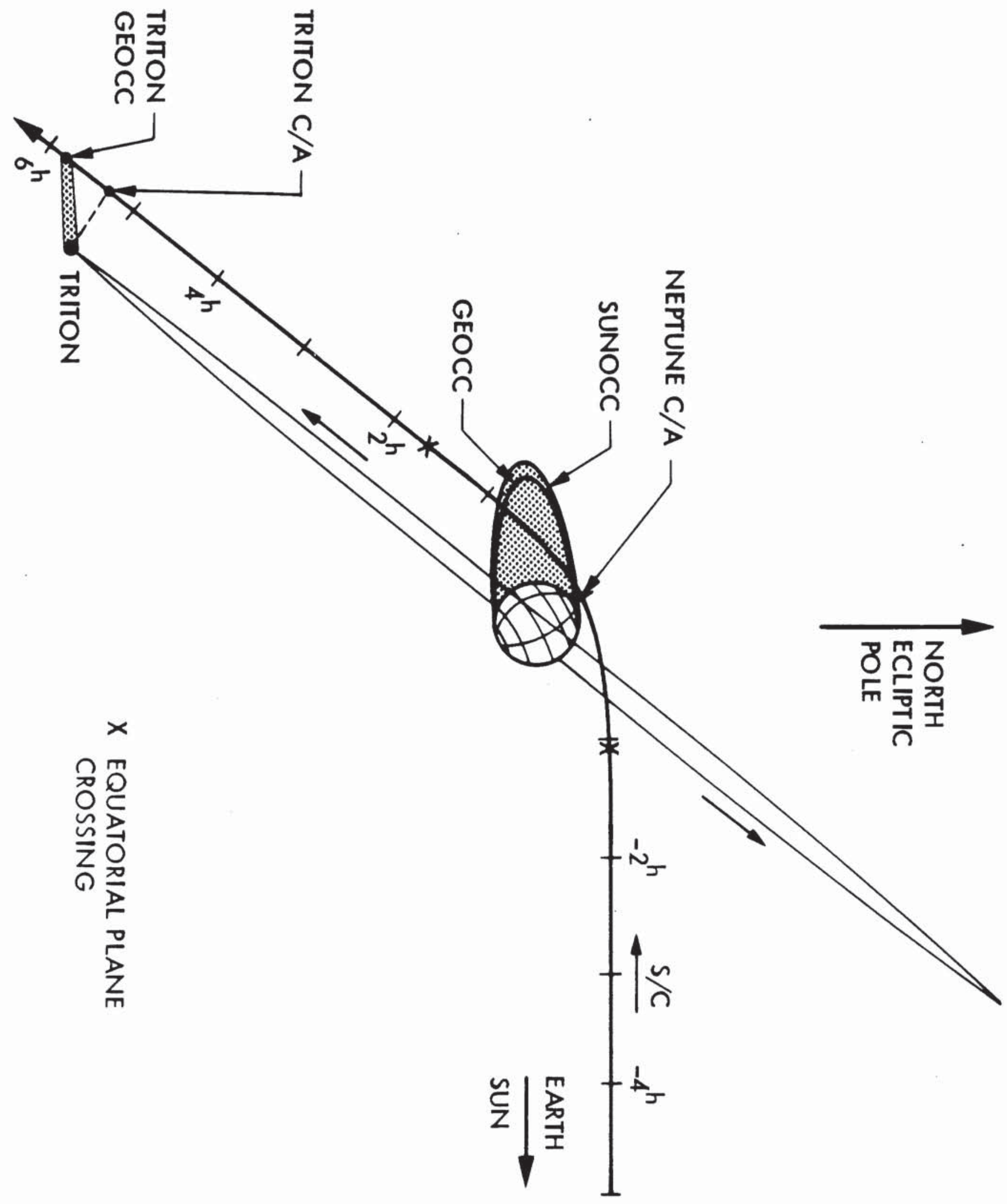

Figure 8 


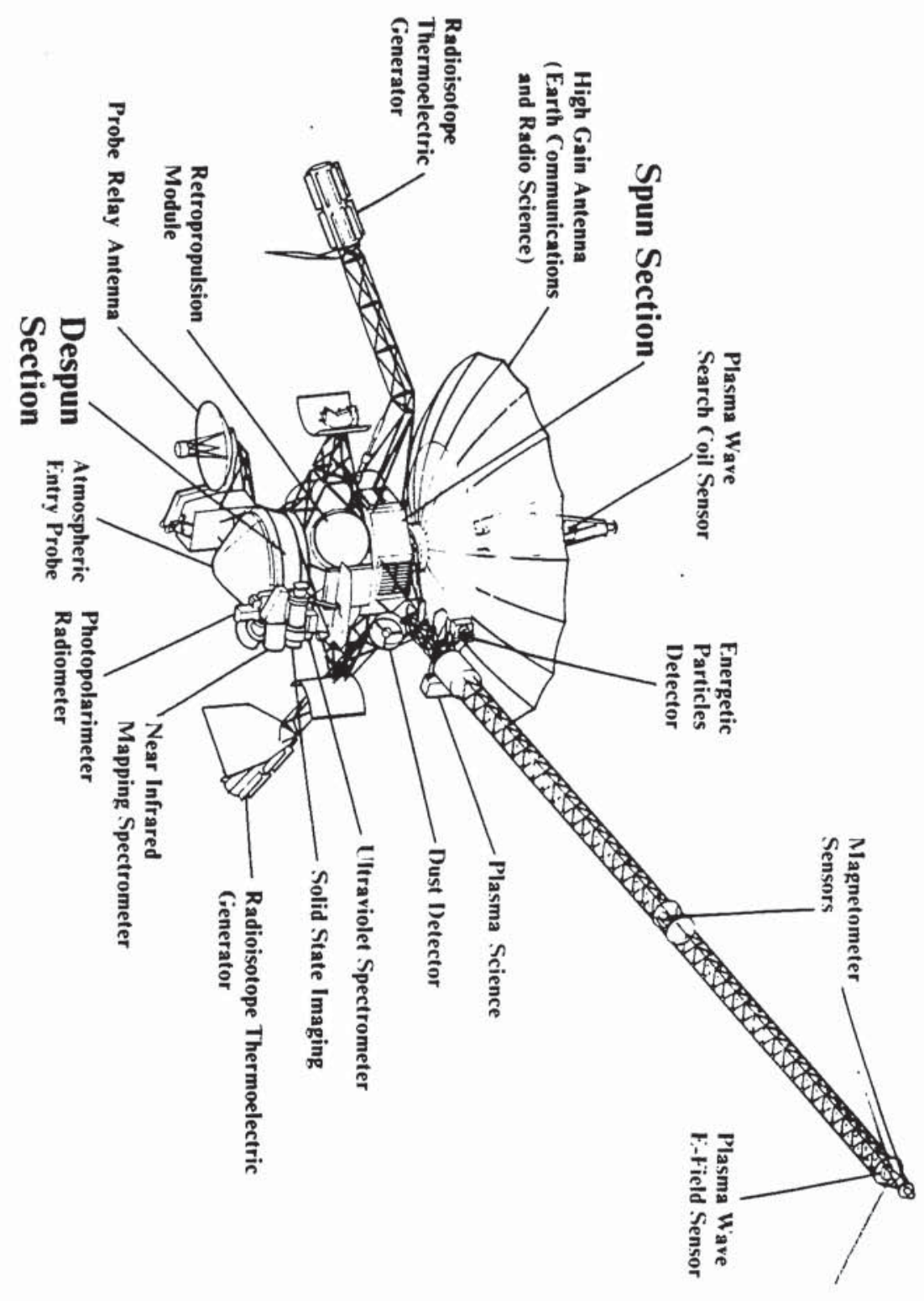

Figure 9 